\title{
Production of conjugated linoleic acid (CLA): effect of inulin on microbial composition and CLA concentration in a human intestinal model
}

\section{Abstract}

Conjugated linoleic acids (CLAs) show a number of putative health-promoting activities including anti-carcinogenic, anti-adipogenic, anti-diabetogenic, anti-inflammatory and antioxidant actions. CLAs are naturally produced by ruminal bacteria and several studies demonstrate that various lactobacilli and bifidobacteria are also able to produce CLAs in vitro from linoleic acid (LA). However, the ability of the human gut microbiota to produce CLA is less extensively studied. Our hypothesis is that the human gut microbiota is able to convert LA to CLA, and that the readily fermentable fiber inulin would positively modulate the growth of CLA-producing bacteria and, consequently increase the CLA content in the intestine.

The capability of the faecal microbiota from five healthy donors to produce CLA was tested in anaerobic batch cultures for 48 hours at $\mathrm{pH} 5.5$ and 6.5. Test treatments were linoleic acid (LA; $1 \mathrm{mg} / \mathrm{mL})+$ bovine serum albumin (BSA; $0.2 \mathrm{mg} / \mathrm{mL})$, and LA $(1 \mathrm{mg} / \mathrm{mL})+$ BSA $(0.2 \mathrm{mg} / \mathrm{mL})+$ inulin $(1 \%, \mathrm{w} / \mathrm{v})$ compared to a control BSA $(0.2 \mathrm{mg} / \mathrm{mL})$ fermentation. The microbial composition was analyzed 0, 24 and 48 hours after starting the fermentation by 16S rRNA gene Illumina MiSeq sequencing (V3-V4 region). CLAs were quantified by Ultra performance liquid chromatography - tandem mass spectrometer (UPLC-MS/MS) and bi-dimensional gas chromatography (GC x GC).

The inclusion of LA + BSA + inulin at pH 5.5 significantly increased the relative abundance of Collinsella aerofaciens $(\mathrm{p}<0.05)$, and tended to increase the relative abundance of bifidobacteria. LA + BSA + inulin at both pH 5.5 and 6.5 reduced the relative abundance of Parabacteroides, Bilophila, Clostridia and Enterobacteriaceae ( $\mathrm{p}<0.05)$. The concentration of CLA, in particular the isomer cis9, trans11 C18:2, was significantly higher in the LA + BSA + inulin group at pH 5.5 after 24 and 48 hours fermentation.

The data show that the treatment LA + BSA + inulin at $\mathrm{pH} 5.5$ induce substantial changes in microbiota composition, including bifidogenesis and CLA production in a human intestinal microbiota model. The changes of relative abundance detected are consistent with changes in gut bacteria previously linked to human health. Collinsella aerofaciens has been reported for reducing bloating, in particular in subjects suffering from irritable bowel syndrome, while Clostridia, Bilophila and Enterobacteriaceae causes human infections. In addition, the increase of bifidobacteria and LAB, which have previously been shown in vitro to produce CLA, may also be involved in CLA production under simulated cecal microbiome. These preclinical observations warrant confirmation in suitably designed animal and human mechanistic studies.

\section{Conflict of Interest}

There is no conflict of interest 